\title{
Cytotoxicity Evaluation of Plant Viral Nanoparticles in HER2+ cell Lines for Breast Cancer Therapy
}

\section{Neda Esfandiari ${ }^{1, *}$}

${ }^{1}$ Protein Research Center, Shahid Beheshti Universtiy, Tehran, Iran

* Corresponding author: Neda Esfandiari, Protein Research Center, Shahid Beheshti

DOI: $10.21859 / \mathrm{mci}$-supp-53 Universtiy, Tehran, Iran, E-mail: neda.esfandiari@yahoo.com

\section{Keywords:}

Plant Viral Nanoparticles

Cytotoxicity

Breast Cancer

HER2+ cells line

MTT assay

\begin{abstract}
Introduction The application of nanotechnology has increased during the last 20 years. Nanoparticles have many favorable properties for applications in medicine. Cancer is the second leading cause of death in the word. Toxicity of nanoparticles especially in medicine is the most important concern. Non-toxic nanoparticles have received considerable attention in recent years. The application of non-toxic Viral Nanoparticles (VNPs) was developed in biomedical imaging and drug delivery. VNPs offer some key advantages: biocompatibility, self-replication, reduce toxicity and cost of treatment. There are many types of viral nanoparticles including VNPs from plants, bacteria and mammals. Since plant viral nanoparticles are less likely to interact with mammals, they are preferred to use in the treatment. In this research, Potato Virus X (PVX) was applied for breast cancer therapy.

Materials and Methods: The toxicity effect of VNPs was evaluated through two cancer cells HER2+. Cells were cultured in RPMI-1640 medium with penicillin-streptomycin $(100 \mathrm{IU} / \mathrm{mL})$ and $10 \% \mathrm{FBS}$ and maintained at $37^{\circ} \mathrm{C}$ in $5 \% \mathrm{CO} 2$ incubator. After $24 \mathrm{~h}$, add $10 \mu \mathrm{g}$ of nanoparticles to cell cultured on 96 -well plate. Cytotoxicity of nanoparticles was investigated by MTT assay. The MTT is used to measuring mitochondrial activity. The viable cells convert MTT into a purple colored product was calculated by absorbance 490540.

Results: The result of this study showed that plant viral nanoparticle was not significantly decreased absorbance level compared to control.

Conclusions: Hence, it seems that this kind of non-toxic nanoparticle, as VNPs, play an important rule in cancer diagnosis and treatment.
\end{abstract}

